\title{
Epífitos vasculares em área com floresta estacional semidecidual, Pelotas, Rio Grande do Sul, Brasil
}

\author{
Vascular epiphytic in an area with mesophilic seasonal forest, Rio Grande do Sul, Brazil
}

\author{
Tângela Denise Perlebergํㅗ Élen Nunes Garcia 2, Sílvia Romeu Pitrez ${ }^{3}$ \\ ${ }^{1}$ Instituto Federal Sul-Rio-Grandense, Pelotas, Rio Grande do Sul, Brasil \\ ${ }^{2}$ Universidade Federal de Pelotas, Pelotas, Rio Grande do Sul, Brasil \\ ${ }^{3}$ Instituto Porto Alegre da Igreja Metodista, Pelotas, Rio Grande do Sul, Brasil
}

\begin{abstract}
Resumo
O presente estudo teve como objetivo conhecer a composição florística de epífitos vasculares de uma Floresta Estacional Semidecidual no interior do município de Pelotas, através de coletas mensais no período de 2009-2012, realizadas a partir de caminhadas aleatórias de forma a abranger toda a área de estudo. No levantamento, foram encontradas 63 espécies, 31 gêneros e nove famílias. Orchidaceae apresentou a maior riqueza, com 24 espécies, seguida por Bromeliaceae com 12, Polypodiaceae com oito, Piperaceae com sete e Cactaceae com cinco. Estas cinco famílias somam $89 \%$ da riqueza de epífitos vasculares na área de estudo. Destacaram-se pela riqueza florística, os gêneros Peperomia e Tillandsia com sete espécies cada, e o gênero Oncidium com seis. Com relação às categorias ecológicas, 59 espécies foram classificadas como holoepífitas habituais, envolvendo a totalidade das espécies de Bromeliaceae, Cactaceae, Orchidaceae, Piperaceae e Polypodiaceae. A maioria das espécies (70\%) ocorreu, simultaneamente, como epífita e como rupícola na floresta em questão. A riqueza epifítica na área em estudo foi considerada alta, levando-se em consideração a posição austral da mata estudada.
\end{abstract}

Palavras-chave: Diversidade, epifitismo, rupícola, região sul do Rio Grande do Sul.

\begin{abstract}
This study aimed to know the floristic composition of vascular epiphytes in a Seasonal Semi-deciduous Forest within the municipality of Pelotas, through monthly collections from 2009 to 2012 made from random walks to cover the entire study area. In the floristic survey, 63 species, 31 genera, and nine families were found. Orchidaceae was the richest, with 24 species, Bromeliaceae with 12, Polypodiaceae with eight, Piperaceae with seven, and Cactaceae with five. These five families account for 89 per cent of vascular epiphytes richness in the study area. Genera Peperomia and Tillandsia stood out for their floristic richness, each one with seven species and genus Oncidium with six. With regard to ecological categories, 59 species were classified as holoepiphytes, involving the total species of Bromeliaceae, Cactaceae, Orchidaceae, Piperaceae, and Polypodiaceae. Most species (70\%) occurred simultaneously as epiphyte and rupicolous in this forest. The epiphytic richness in the study area was considered high taking into account the southern position of the forest studied.
\end{abstract}

Keywords: Diversity, epiphytism, rupicolous, southern Rio Grande do Sul State 


\section{Introdução}

A metade sul do Rio Grande do Sul, correspondente ao Bioma Pampa (IBGE, 2004), é a porção de maior diversificação topográfica e geológica da Região Sul do Brasil (AB'SÁBER, 2005). A Encosta do Sudeste encontra-se nesta porção, abrangendo as encostas leste das serras do Herval e dos Tapes (FORTES, 1959). Encontrando-se bastante degradada, mas ainda pouco estudada, a cobertura vegetal da Encosta do Sudeste, segundo Teixeira et al. (1986), constitui-se de Floresta Estacional Semidecidual, a qual é marcada pelo fenômeno da estacionalidade, além da semidecidualidade foliar que atinge cerca de 20 a 50\% do estrato superior da floresta. No Rio Grande do Sul, em específico, a semidecidualidade está diretamente relacionada à amplitude anual de temperatura (TEIXEIRA et al., 1986).

As regiões oeste e noroeste do município de Pelotas fazem parte da Encosta do Sudeste e, segundo Rosa (1985), no passado, eram cobertas por Floresta Estacional Semidecidual. Atualmente, estas florestas encontram- se bastante fragmentadas devido à retirada seletiva de madeira e uso do fogo, além da expansão da agricultura e das pastagens. Persistem, no entanto, algumas áreas com vegetação bem conservada em função, principalmente, da acidentada topografia. Nestes remanescentes de floresta, pode-se observar uma considerável flora epifítica que, em muitas ocasiões, compartilha espécies com comunidades que se desenvolvem sobre rochas presentes no interior destas florestas.

As epífitas representam $10 \%$ de toda flora vascular mundial e estão distribuídas em 84 famílias, sendo mais abundantes nos Neotrópicos, onde constituem 50\% de toda a flora vascular (KRESS, 1986; BENZING, 1990). O Brasil conta com 49 famílias que possuem representantes epifíticos, a maioria monocotiledôneas (66\%), principalmente Orchidaceae (50\%), Bromeliaceae (12\%) e Araceae (4\%) (KERSTEN, 2010).

Atualmente, vários são os trabalhos realizados no Brasil com a flora epifítica. Abordam, principalmente, a composição florística e aspectos ecológicos desta sinúsia, e a grande maioria deles concentra-se nas regiões sul e sudeste, nas mais diferentes formações vegetais. No Paraná, diversos estudos com epífitos foram realizados, citando-se para formações vegetais com Floresta Estacional Semidecidual os de Borgo et al. (2002), Cervi e Borgo (2007), Dettke et al. (2008) e Geraldino et al. (2010).

No Rio Grande do Sul, os trabalhos sobre a flora epifítica concentram-se principalmente no norte da região fisiográfica do Litoral (WAECHTER, 1986, 1992, 1998; BREIER, 1999; GONÇALVES e WAECHTER,
2003; ROGALSKI e ZANIN, 2003; GIONGO e WAECHTER, 2004; BUZATTO et al. 2008). Na região sul do estado, principalmente no município de Pelotas, estudos específicos relacionados à totalidade desta sinúsia inexistem na literatura, apenas trabalhos que se concentram em uma ou poucas famílias botânicas (PERLEBERG e TOMKOWSKI, 2007; PERLEBERG et al., 2008).

Este trabalho teve como objetivo conhecer a composição florística de epífitos vasculares em uma área de Floresta Estacional Semidecidual no interior do município de Pelotas, Rio Grande do Sul.

\section{Material e Métodos}

\section{1 Área de estudo}

O estudo foi realizado em uma área com Floresta Estacional Semidecidual Sub-montana (TEIXEIRA et al., 1986), na localidade de Colônia Triunfo, interior do município de Pelotas, sul do Rio Grande do Sul (31 $22^{\prime} 54^{\prime \prime}$ S e 52²9'26" W). Situada na região fisiográfica da Encosta do Sudeste (FORTES, 1959), a zona rural do município de Pelotas apresenta morros de ondulações moderadas, com altitudes predominantes inferiores a 100 metros, podendo chegar a 300 metros nos locais mais elevados (ROSA, 1985).

O clima da região é do tipo Cfa, segundo classificação de Köppen, subtropical úmido, sem estiagens, com temperaturas médias anuais entre $13,8^{\circ}$ e $22,9^{\circ} \mathrm{C}$. Janeiro apresenta média de $23,2^{\circ} \mathrm{C}$, sendo o mês mais quente, e julho, com média de $12,3^{\circ} \mathrm{C}$, o mês mais frio (MOTA, 1951). A precipitação pluviométrica média anual é de $1.366,9 \mathrm{~mm}$, com chuvas regularmente distribuídas durante todo o ano, sendo fevereiro o mês mais chuvoso, com média de 153,3mm (SCHÖFFEL et al., 2013). Os solos predominantes são do tipo Argissolo Amarelo Distrófico Câmbico, que apresentam uma percentagem considerável de afloramentos rochosos (CUNHA e SILVEIRA, 1996).

A floresta estudada apresenta cerca de três hectares em bom estado de conservação, o que se deve, provavelmente, a presença de grandes matacões, tornando-a uma área imprópria para uso na agricultura. Concentra-se na encosta oeste de um morro, localizado em uma propriedade particular. No entorno da área, existem plantios de eucaliptos, pequenas lavouras e fragmentos de floresta em diversos estágios de regeneração. Não existem cursos de água no interior da floresta. O curso de água mais próximo, um pequeno arroio, circunda a base do morro.

\subsection{Levantamento florístico e análise dos dados}


As coletas de material botânico foram mensais, realizadas a partir de caminhadas aleatórias por toda a área de estudo, e ocorreram no período de janeiro 2009 a fevereiro de 2012, totalizando cerca de 50 visitas. As espécies foram registradas, fotografadas, recolhidas quando férteis e levadas ao herbário PEL, do Departamento de Botânica da Universidade Federal de Pelotas, para identificação, herborização e inclusão no referido herbário. Devido ao grande número, as espécies rupícolas também foram identificadas neste estudo. Para a identificação das espécies, foi utilizada literatura especializada para cada família, comparação com o material de herbário e, quando necessário, especialistas foram consultados.

As angiospermas foram organizadas segundo o sistema APG III (ANGIOSPERM PHYLOGENY GROUP, 2009) e as pteridófitas segundo Smith et al. (2006). As abreviaturas de autores seguem o Index Kewensis on line (2013).

As espécies levantadas foram classificadas em categorias ecológicas, conforme sua relação com o forófito de acordo com Benzing (1990) em: holoepífitos característicos (HLC), holoepífitos facultativos (HLF) e holoepífitos acidentais (HLA). Também foi feito o registro das espécies rupícolas, como aquelas plantas encontradas sobre as superfícies rochosas, tanto nos locais com maior ou menor inclinação da rocha, desconsiderando-se apenas as plantas estabelecidas sobre depósitos de sedimentos ou, propriamente, sobre solos.

\section{Resultados e discussão}

Foram registradas 63 espécies de epífitos vasculares na área de estudo, distribuídas em 31 gêneros e nove famílias - 13 espécies de pteridófitas e 50 espécies de angiospermas (Tab. 1). As famílias com maior riqueza florística foram: Orchidaceae com 24 espécies (38\%), Bromeliaceae com 12 espécies (19\%), Polypodiaceae com oito espécies (13\%), Piperaceae com sete espécies (11\%) e Cactaceae com cinco espécies (8\%). Juntas, estas famílias somam $89 \%$ do total das espécies epífitas na área de estudo. Estes dados seguem o padrão encontrado na maioria dos levantamentos realizados com epífitas na região Sul do Brasil, onde apenas cinco famílias (Orchidaceae, Bromeliaceae, Polypodiaceae, Piperaceae e Cactaceae) detêm mais de $70 \%$ das espécies e a família Orchidaceae representa mais de 30\% das espécies desta sinúsia (GERALDINO et al. 2010; BUZATTO et al., 2008; BORGO e SILVA, 2003; GONÇALVES e WAECHTER, 2003; ROGALSKI e ZANIN, 2003; KERSTEN e SILVA, 2001, 2002; BREIER, 1999; DITTRICH et al., 1999; WAECHTER, 1992; 1998). Destacaram-se pela riqueza florística os gêneros Peperomia e Tillandsia, com sete espécies cada, e o gênero Oncidium, com seis espécies (Tab. 1).

Com relação às categorias ecológicas (Tab. 1), 44 espécies $(70 \%)$ foram encontradas crescendo tanto sobre árvores quanto sobre rochas, portanto foram classificadas como holoepífitas facultativas. A totalidade de espécies das famílias Aspleniaceae, Cactaceae, Gesneriaceae e Piperaceae encontram-se nesta categoria ecológica. Sinningia macrostachya, espécie comumente citada na literatura como sendo rupícola (GRELA e BRUSSA, 2005), na mata em estudo, foi encontrada como epífita desenvolvendo-se sobre fustes de árvores a pleno sol. Apenas Rumohra adiantiformis e Asplenium claussenii foram consideradas como sendo holoepífitas acidentais por serem frequentemente encontradas como terrícolas. Na categoria ecológica holoepífitos característicos, registrou-se a ocorrência de 17 espécies (27\%) pertencentes às famílias Bromeliaceae, Orchidaceae e Polypodiaceae, as quais foram encontradas vegetando exclusivamente sobre outros vegetais (Tab. 1).

Tabela 1. Epífitos vasculares em área com Floresta Estacional Semidecidual, Pelotas, Rio Grande do Sul, Brasil (entre 2009 e 2012), com suas respectivas categorias ecológicas (CE): HLC - holoepífita característica, HLF - holoepífita facultativa, HLA - holoepífita acidental; RUP - espécies que foram encontradas sobre rochas; Voucher.

\begin{tabular}{clll}
\hline Família/Espécies & CE & RUP & Vouchers \\
\hline Aspleniaceae & & & \\
\hline Asplenium claussenii Hieron & HLA & $\mathrm{X}$ & T. Perleberg 108 \\
\hline Asplenium scandicinum Kaulf. & HLF & $\mathrm{X}$ & T. Perleberg 112 \\
\hline Asplenium incurvatum Fée & HLF & $\mathrm{X}$ & T. Perleberg 070 \\
\hline
\end{tabular}


Tabela 1. Continuação...

\begin{tabular}{|c|c|c|c|}
\hline Família/Espécies & $\mathbf{C E}$ & RUP & Vouchers \\
\hline \multicolumn{4}{|l|}{ Bromeliaceae } \\
\hline Aechmea recurvata (Klotzsch) L.B. Sm. & HLF & $\mathrm{X}$ & T. Perleberg 033 \\
\hline Billbergia nutans H.Wendl. & HLF & $\mathrm{X}$ & T. Perleberg 142 \\
\hline Tillandsia aeranthos (Loisel.) L.B. Sm. & HLC & & T. Perleberg 010 \\
\hline Tillandsia geminiflora Brongn. & HLF & $\mathrm{X}$ & T. Perleberg 023 \\
\hline Tillandsia mallemontii Glaz. ex Mez & HLC & & T. Perleberg 064 \\
\hline Tillandsia stricta Sol. ex Sims & HLF & $\mathrm{X}$ & T. Perleberg 049 \\
\hline Tillandsia recurvata (L.) L. & HLC & & T. Perleberg 017 \\
\hline Tillandsia tenuifolia $\mathrm{L}$. & HLF & $\mathrm{X}$ & T. Perleberg 082 \\
\hline Tillandsia usneoides (L.) L. & HLC & & T. Perleberg 139 \\
\hline Vriesea friburgensis $\mathrm{Mez}$ & HLF & $\mathrm{X}$ & T. Perleberg 044 \\
\hline Vriesea gigantea Gaudich. & HLF & $\mathrm{X}$ & T. Perleberg 323 \\
\hline Vriesea platynema Gaudich. & HLF & $\mathrm{X}$ & T. Perleberg 029 \\
\hline
\end{tabular}

\section{Cactaceae}

\begin{tabular}{clll}
\hline Lepismium cruciforme (Vell.) Miq. & HLF & $\mathrm{X}$ & T. Perleberg 013 \\
\hline $\begin{array}{c}\text { Lepismium warmingianum (Schum.) } \\
\text { Barthlott }\end{array}$ & HLF & $\mathrm{X}$ & T. Perleberg 037 \\
\hline Rhipsalis cereuscula Haw. & HLF & $\mathrm{X}$ & T. Perleberg 103 \\
\hline Rhipsalis floccosa Salm-Dyck ex Pfeiff. & HLF & $\mathrm{X}$ & T. Perleberg 102 \\
\hline Rhipsalis teres Steud. & HLF & $\mathrm{X}$ & T. Perleberg 077 \\
\hline
\end{tabular}

Dryopteridaceae

\begin{tabular}{llll}
\hline Rumohra adiantiformis (G.Forst.) Ching & HLA & X & T. Perleberg 012 \\
\hline
\end{tabular}

\section{Gesneriaceae}

\begin{tabular}{clll}
\hline Sinningia douglasii (Lindl.) Chautems & HLF & $\mathrm{X}$ & T. Perleberg 155 \\
\hline $\begin{array}{c}\text { Sinningia macrostachya (Lindl.) Chau- } \\
\text { tems }\end{array}$ & HLF & $\mathrm{X}$ & T. Perleberg 031 \\
\hline
\end{tabular}

\section{Orchidaceae}

\begin{tabular}{cccc}
\hline $\begin{array}{c}\text { Acianthera hygrophila (Barb.Rodr.) } \\
\text { Pridgeon \& M.W.Chase }\end{array}$ & HLF & $\mathrm{X}$ & T. Perleberg 325 \\
\hline $\begin{array}{c}\text { Acianthera saurocephala } \text { (Lodd.) } \\
\text { Pridgeon \& M.W.Chase }\end{array}$ & HLF & $\mathrm{X}$ & T. Perleberg 147 \\
\hline $\begin{array}{c}\text { Acianthera sonderana (Rchb.f) Pridgeon } \\
\text { \& M.W.Chase }\end{array}$ & HLC & A. & Thiel 093 \\
\hline
\end{tabular}


Tabela 1. Continuação...

\begin{tabular}{|c|c|c|c|}
\hline Família/Espécies & $\mathbf{C E}$ & RUP & Vouchers \\
\hline $\begin{array}{l}\text { Anathallis malmeana (Dutra ex Pabst) } \\
\text { Pridgeon \& M.W.Chase }\end{array}$ & HLF & $\mathrm{X}$ & T. Perleberg 156 \\
\hline $\begin{array}{c}\text { Anathallis obovata (Lindl.) Pridgeon \& } \\
\text { M.W.Chase }\end{array}$ & HLF & $\mathrm{X}$ & T. Perleberg 094 \\
\hline Barbosella australis Schltr. & HLF & $\mathrm{X}$ & T. Perleberg 110 \\
\hline $\begin{array}{l}\text { Brasiliorchis porphyrostele (Rchb.f.) } \\
\text { R.B.Singer, S.Koehler \& Carnevali }\end{array}$ & HLF & $\mathrm{X}$ & T. Perleberg 324 \\
\hline Campylocentrum aromaticum Barb.Rodr. & $\mathrm{HLC}$ & & T. Perleberg 041 \\
\hline Dryadella zebrina (Porsch) Luer & HLC & & T. Perleberg 326 \\
\hline Eurystyles lorenzii (Cogn.) Schltr. & HLC & & T. Perleberg 166 \\
\hline $\begin{array}{c}\text { Isabelia pulchella (Kraenzl.) Senghas \& } \\
\text { Teusch. }\end{array}$ & HLC & & T. Perleberg 005 \\
\hline Octomeria diaphana Lindl. & HLF & $\mathrm{X}$ & T. Perleberg 100 \\
\hline Octomeria umbonulata Schltr. & HLC & & T. Perleberg 327 \\
\hline Oncidium bifolium Sims & HLF & $\mathrm{X}$ & T. Perleberg 329 \\
\hline Oncidium fimbriatum Lindl. & HLF & $\mathrm{X}$ & T. Perleberg 330 \\
\hline Oncidium concolor Hook. & HLC & & T. Perleberg 328 \\
\hline Oncidium flexuosum Sims & HLF & $\mathrm{X}$ & T. Perleberg 159 \\
\hline Oncidium hians Lindl. & HLF & $\mathrm{X}$ & T. Perleberg 007 \\
\hline Oncidium longipes Lindl. & HLF & $\mathrm{X}$ & T. Perleberg 001 \\
\hline Platyrhiza quadricolor Barb. Rodr. & HLC & & T. Perleberg 042 \\
\hline $\begin{array}{l}\text { Specklinia marginalis (Rchb. f.) F. Bar- } \\
\text { ros }\end{array}$ & HLF & $\mathrm{X}$ & T. Perleberg 331 \\
\hline $\begin{array}{l}\text { Specklinia seriata (Lindl.) Pridgeon \& } \\
\text { M.W.Chase }\end{array}$ & HLF & $\mathrm{X}$ & T. Perleberg 332 \\
\hline Stelis papaquerensis Rchb.f. & HLF & $\mathrm{X}$ & T. Perleberg 011 \\
\hline $\begin{array}{l}\text { Trichocentrum pumilum (Lindl.) M.W. } \\
\text { Chase \& N.H.Williams }\end{array}$ & HLC & & T. Perleberg 333 \\
\hline
\end{tabular}

Piperaceae

\begin{tabular}{clll}
\hline Peperomia catharinae Miq. & HLF & $\mathrm{X}$ & T. Perleberg 036 \\
\hline Peperomia glabella A.Dietr. & HLF & $\mathrm{X}$ & T. Perleberg 043 \\
\hline Peperomia corcovadensis Gardner & HLF & $\mathrm{X}$ & T. Perleberg 065 \\
\hline Peperomia delicatula Hench. & HLF & $\mathrm{X}$ & T. Perleberg 035 \\
\hline Peperomia psilostachya C.DC. & HLF & $\mathrm{X}$ & T. Perleberg 104 \\
\hline Peperomia tetraphylla Hook. \& Arn. & HLF & $\mathrm{X}$ & T. Perleberg 006 \\
\hline Peperomia urocarpa Fisch. \& C.A.Mey. & HLF & $\mathrm{X}$ & T. Perleberg 133 \\
\hline
\end{tabular}


Tabela 1. Continuação...

\begin{tabular}{|c|c|c|c|}
\hline Família/Espécies & $\mathbf{C E}$ & RUP & Vouchers \\
\hline \multicolumn{4}{|l|}{ Polypodiaceae } \\
\hline Campyloneurum nitidum C.Presl & HLF & $\mathrm{X}$ & T. Perleberg 003 \\
\hline $\begin{array}{c}\text { Microgramma squamulosa (Kaulf.) de } \\
\text { la Sota }\end{array}$ & HLC & & T. Perleberg 066 \\
\hline $\begin{array}{l}\text { Microgramma vacciniifolia (Langsd. \& } \\
\text { Fisch.) Copel. }\end{array}$ & HLC & & T. Perleberg 067 \\
\hline Niphidium rufosquamatum Lellinger & HLF & $\mathrm{X}$ & T. Perleberg 056 \\
\hline $\begin{array}{l}\text { Pecluma pectinatiformis (Lindm.) } \\
\text { M.G.Price }\end{array}$ & HLF & $\mathrm{X}$ & T. Perleberg 334 \\
\hline Pleopeltis pleopeltifolia (Raddi) Alston & HLC & & T. Perleberg 058 \\
\hline Polypodium hirsutissimum Raddi & HLF & $\mathrm{X}$ & T. Perleberg 032 \\
\hline
\end{tabular}

Pteridaceae

\begin{tabular}{llll}
\hline Vittaria lineata $(\mathrm{L}$.$) Sm.$ & HLF & X & T. Perleberg 057 \\
\hline
\end{tabular}

Estes resultados indicam que a presença de matacões com diferentes tamanhos e conformações no interior da floresta representa maior diversidade de microhabitats para colonização, tanto por espécies epífitas quanto terrícolas, que ali se estabelecem e se reproduzem com sucesso semelhante.

As rochas maiores formam clareiras na floresta, recebendo luminosidade suficiente para que as espécies mais exigentes neste parâmetro possam povoá-las. Por outro lado, estas rochas oferecem, em suas laterais bem sombreadas, microhabitats para que outras espécies ali se desenvolvam. Os matacões menores que, por sua vez, ficam sombreados, disponibilizam ambientes para o desenvolvimento de espécies que preferem crescer à sombra e com maior umidade. Nas rochas onde o topo fica exposto à radiação solar direta foram encontradas com maior frequência Aechmea recurvata, Rumohra adiantiformis, Sinningia macrostachya e Sinningia douglasii; enquanto que Billbergia nutans, Octomeria diaphana e espécies de Peperomia foram encontradas nas áreas sombreadas, onde a umidade é maior, geralmente nas laterais ou nas rochas menores sombreadas pelas árvores. Specklinia marginalis e Barbosella australis foram observadas formando verdadeiros tapetes sobre as rochas, cobrindo boa parte de suas laterais.

Esta distribuição das plantas sobre as rochas parece seguir o mesmo padrão de distribuição observado nas epífitas sobre os forófitos: na copa das árvores, onde a incidência luminosa é maior, desenvolvem-se as espécies mais exigentes em luminosidade, Oncidium flexuosum, Oncidium longicornu, Aechmea recurvata, entre outras, enquanto nos fustes, onde a luminosidade é menor desenvolvem-se as espécies de Peperomia, Oncidium fimbriatum, Billbergia nutans, entre outras. Outra característica observada refere-se à modificação no porte e na coloração das plantas, independente do substrato onde ocorrem em decorrência da maior ou menor exposição ao sol. Aechmea recurvata, Brasiliorchis porphyrostelle e Oncidium flexuosum, em lugares mais ensolarados, apresentaram folhas mais curtas e com coloração verde-amarelada; nos lugares sombreados, as folhas apresentaram-se mais longas e de um verde mais intenso.

Ao se comparar este estudo com outros realizados em Florestas Estacionais Semideciduais, na região Sul e Sudeste do Brasil, o número de espécies foi superior (Tab. 2). No entanto, deve-se considerar que há diferenças entre as áreas amostradas, as metodologias empregadas, além do estado de conservação das florestas, que está baseado no resultado dos tipos de usos e impactos particulares de cada região. Dettke et al. (2008) realizaram o estudo em floresta alterada no município de Maringá (PR) e Cervi e Borgo (2007) fizeram um levantamento preliminar no Parque Nacional do Iguaçu (PR). A riqueza foi semelhante ao estudo realizado por Geraldino et al. (2010), os quais encontraram 61 espécies em área com Floresta Estacional Semidecidual (Tab. 2). 
Tabela 2. Riqueza de epífitos vasculares em Florestas Estacionais Semideciduais (FES) na Região Sul e Sudeste do Brasil, organizado por ordem decrescente de riqueza.

\begin{tabular}{cccc}
\hline Localidade & Formação Vegetal & $\mathbf{N}^{\mathbf{0}}$ de espécies & Fonte \\
\hline Pelotas, RS & FES & 63 & Presente estudo \\
$\begin{array}{c}\text { Capela do Calvário, Campo } \\
\text { Mourão, PR }\end{array}$ & FES & 61 & Geraldino et al. (2010) \\
Parque Nacional do Iguaçu, PR & FES & 56 & Cervi e Borgo (2007) \\
Viamão, RS & FES & 54 & Breier (1999) \\
Reserva CUASO, São Paulo, SP & FES & 37 & Dislich e Mantovani (1998) \\
PEVR, Fênix, PR & FES & 32 & Borgo et al. (2002) \\
Parque do Ingá, Maringá, PR & FES & 29 & Dettke et al. (2008) \\
Reserva CUASO, São Paulo, SP & FES & 25 & Breier (2005) \\
\hline
\end{tabular}

No Rio Grande do Sul, a maioria dos estudos realizados com epífitos concentra-se ao norte do paralelo 30, considerado como limite sul para a ocorrência de muitos táxons epifíticos tropicais, em decorrência da mudança na exposição da encosta atlântica de oceânica para continental (WAECHTER, 1992). No Litoral do estado, Waechter (1992) demonstrou que o contingente epifítico diminui do norte para o sul ao comparar duas formações distantes latitudinalmente: Torres, ao norte, com 93 espécies de epífitos e Taim, ao sul, com 24 espécies. Portanto, considerando-se a posição austral da floresta estudada, a sua riqueza epifítica é alta, o que se deve, certamente, ao estado de conservação da mesma.

Algumas espécies como Cattleya intermedia Graham, Oncidium ciliatum Lindl. e Acianthera pubescens (Lindl.) Pridgeon \& M.W.Chase, pertencentes à Orchidaceae, bem como Rhipsalis lumbricoides Lem. (Cactaceae), que são comumente encontradas nas florestas de restinga presentes na região de planície do município de Pelotas (PERLEBERG e TOMKOWSKI, 2007; PERLEBERG et al., 2008), não foram observadas na floresta avaliada. Da mesma forma, algumas espécies registradas neste estudo (Oncidium bifolium, Oncidium concolor, Oncidium hians, Vriesea platynema, entre outras) não são observadas nas florestas de restinga da região. Estas observações indicam a existência de grupos florísticos exclusivos das formações vegetacionais que ocupam diferentes altitudes (planície e serra do sudeste). Mas os estudos existentes ainda são escassos para que se façam quaisquer delimitações. Os resultados obtidos demonstram que o conhecimento da flora epifítica, sob o ponto de vista florístico e fitogeográfico, são ainda insuficiente no Rio Grande do Sul, principalmente no que se refere ao domínio da Floresta Estacional Semidecidual da região Sul do Estado.

\section{Conclusão}

A mata estudada apresenta grande riqueza de epífitos vasculares, levando-se em consideração a posição austral da mesma. Esta grande riqueza pode estar associada ao grau de conservação da mata estudada. Portanto, pode-se concluir que pequenos remanescentes de mata abrigam, se não a totalidade, uma grande parcela da riqueza da flora existente na região. Daí a importância do conhecimento e da conservação destes remanescentes.

\section{Referências}

AB'SÁBER, A. N. Os domínios da natureza no Brasil. Potencialidades paisagísticas. Cutia: Ateliê Editorial, 2005. 159p.

ANGIOSPERM PHYLOGENY GROUP. An update of the Angiosperm Phylogeny Group classification 
for the orders and families of flowering plants: APG III. Botanical Journal of the Linnaean Society, v.161, p.105-121, 2009.

BENZING, D. H. Vascular epiphytes. General biology and related biota. Cambridge: Cambridge University Press, 1990. 354p.

BORGO, M.; SILVA, S. M.; PETEAN, M. P. Epífitos vasculares em um remanescente de floresta estacional semidecidual, município de Fênix, PR, Brasil. Acta Biologica Leopoldensia, v.24, p.121130, 2002.

BORGO, M.; SILVA, S. M. Epífitos vasculares em fragmentos de Floresta Ombrófila Mista, Curitiba, Paraná, Brasil. Revista Brasileira de Botânica, v.26, p.391-401, 2003.

Breier, T. B. Florística e ecologia de epífitos vasculares em uma floresta costeira do sul do Brasil. 1999. 83 f. Dissertação (Mestrado em Botânica) - Universidade Federal do Rio Grande do Sul, Porto Alegre.

BREIER, T. B. O epifitismo vascular em florestas do sudeste do Brasil. 2005. 139 f. Tese (Doutorado em Biologia Vegetal) - Universidade Estadual de Campinas, Campinas.

BUZATTO, C. R.; SEVERO, B. M. A.; WAECHTER, J. L. Composição florística e distribuição ecológica de epífitos vasculares na Floresta Nacional de Passo Fundo, Rio Grande do Sul. Iheringia, v.63, p.231-239, 2008.

CERVI, A. C.; BORGO M. Epífitos vasculares no Parque Nacional do Iguaçu, Paraná (Brasil). Levantamento preliminar. Fontqueria, v.55(51), p.415-422, 2007.

Cunha, N. G.; R. J. C. Silveira. Estudo dos solos do município de Pelotas. Pelotas: EMBRAPA/CPACT, 1996. $54 \mathrm{p}$.

DETTKE, G. A.; ORFRINI, A. C.; MILANEZEGUTIERRE, M. A. Composição florística e distribuição de epífitas vasculares em um remanescente alterado de Floresta Estacional Semidecidual no Paraná, Brasil. Rodriguésia, v.59, p.859-872, 2008.

DISLICH, R.; MANTOVANI, W. A flora de epífitas da reserva da Cidade Universitária "Armando de Salles Oliveira" (São Paulo, Brasil). Boletim Botânico da Universidade de São Paulo, v.17, p.6184, 1998.

DITTRICH, V. A. O.; KOZERA, C.; MENEZESSILVA, S. Levantamento florístico dos epífitos vasculares do Parque Bariguí,Curitiba, Paraná, Brasil. Theringia, v.52, p.11-22, 1999.

FORTES, A. B. Geografia física do Rio Grande do Sul. Porto Alegre: Globo, 1959. 393 p.

GERALDINO, H. C. L.; CAXAMBÚ, M. G.; SOUZA, D. C. Composição florística e estrutura da comunidade de epífitas vasculares em uma área de ecótono em Campo Mourão, PR, Brasil. Acta Botânica Brasílica, v.24, p.469-482, 2010.

GIONGO, C.; WAECHTER, J. L. Composição florística e estrutura comunitária de epífitos vasculares em uma floresta de galeria na Depressão Central do Rio Grande do Sul. Revista Brasileira de Botânica, v.3, p.567-572; 2004.

Gonçalves, N. C.; WAECHTER, J. L. Aspectos florísticos e ecológicos de epífitos vasculares sobre figueiras isoladas no norte da planície costeira do Rio Grande do Sul. Acta Botânica Brasílica, v.17, p.89-100, 2003.

GRELA, I. A.; BRUSSA, C. A. Sinningia macrostachya (Lindl.) Chautems, nuevo registro de Gesneriaceae para La flora Del Uruguay. Iheringia, v.60, p.249-252, 2005.

IBGE. Mapa de Biomas do Brasil. Escala 1:5.000.000. 2004. Disponível em: <http://mapas. ibge.gov.br/tematicos>. Acesso em: mar. 2013.

INDEX KEWENSIS. The International Plant Names Index. 2013. Disponível em: $<\mathrm{http}: / / \mathrm{www}$. ipni.org/ipni/plantnamesearchpage.do $>$. Acesso em: mar. 2013.

KERSTEN, R. A. Epífitas vasculares. Histórico, participação taxonômica e aspectos relevantes, com ênfase na Mata Atlântica. Hoehnea, v.37, p.938, 2010.

KERSTEN, R. A.; SILVA, S. M. Composição florística e estrutura do componente epifítico vascular em floresta da planície litorânea na Ilha do Mel, Paraná, Brasil. Revista Brasileira de Botânica, v.24, p.213-226, 2001. 
KERSTEN, R. A.; SILVA, S. M. Florística e estrutura do componente epifítico vascular em floresta ombrófila mista aluvial do Rio Barigui, Paraná, Brasil. Revista Brasileira de Botânica, v.25, p.259-267, 2002.

KRESS, W. J. The systematic distribution of vascular epiphytes: an update. Selbyana, v.9, p.222, 1986.

MOTA, F. S. Estudos do clima do estado do Rio Grande do Sul, segundo o sistema de W. Köppen. Revista Brasileira de Geografia, v.2, p.275-284, 1951.

PERLEBERG, T. D.; GOMES, J. C. C.; VARGAS, D. M. O gênero Oncidium Sw. (Orchidaceae) no sul do Rio Grande do Sul, Brasil. BioScriba, v.1, p.7679, 2008.

PERLEBERG, T. D.; TOMKOWSKI, P. B. P. Bromeliaceae e Orchidaceae epífitas nas trilhas do Ecocamping Municipal de Pelotas, RS, Brasil. Revista Brasileira de Biociências, v.5, p.720-722, 2007.

Rogalski, J. M.; Zanin, E. M. Composição florística de epífitos vasculares no estreito de Augusto César, Floresta Estacional Decidual do Rio Uruguai, RS, Brasil. Revista Brasileira de Botânica, v.26, p.551556, 2003.

Rosa, M. Geografia de Pelotas. Pelotas: Editora da Universidade Federal de Pelotas. 1985, 333 p.

SCHÖFFEL, E. R.; STEINMETZ, S.; LUZ, L. O. F.; SILVA, P. R. G.; MIORI, P. R. B. Estação Agroclimatológica de Pelotas. 2013. Disponível em: <http://www.cpact.embrapa.br/agromet/estacao/ creditos.html>. Acesso em: abr. de 2013.

SMITH, A. R.; PRYER, M. K.; SCHUETTPELZ, E.; KORALL, P.; SCHNEIDER, H.; WOLF, P. G. A classification for extant ferns. Táxon, v.55, p.705731, 2006.

TEIXEIRA, M. B.; COURA NETO, A. B.; PASTORE, U.; RANGEL FILHO, A. L. R. Vegetação. In: Levantamento dos recursos naturais. Rio de Janeiro: IBGE, v.33, p.541-632, 1986.

Waechter J. L. Epífitos vasculares da mata paludosa do Faxinal, Torres, Rio Grande do Sul, Brasil. Iheringia, v.34, p.39-49, 1986.
Waechter, J. L. O epifitismo vascular na planície costeira do Rio Grande do Sul. 1992. 163 f. Tese (Doutorado) - Universidade Federal de São Carlos, São Carlos.

Waechter, J. L. Epifitismo vascular em uma floresta de restinga do Brasil subtropical. Ciência e Natura, v.20, p.43-66, 1998. 